\title{
Titulares, hashtags y videojuegos
}

\section{POR ADRIANA AMADO}

amadoa@catedraa.com.ar - Facultad Latinoamericana de Ciencias Sociales (FLACSO), Argentina.

El libro Titulares, hashtags y videojuegos no es un libro. O no solo eso. Desde su funcionalidad como publicación compila las participaciones en el primer congreso del Centro de Estudios sobre Medios y Sociedad (MESO), un espacio de investigación y debate en la Universidad de San Andrés (UDESA). Desde su valor simbólico, el libro consagra la instancia de creación de ese espacio en 2015 , que significó un movimiento sutil pero consistente en el campo de la comunicación de la Argentina. Y no porque no existiera una larga tradición en el país de actividades académicas y referencias consagradas en el siglo XX, sino porque ese espacio vino a proponer una apertura hacia temas y métodos de estudio del siglo XXI.

El enlace de sus directores, Eugenia Mitchelstein por la UDESA y Pablo Boczkowski por la Northwestern University (a la vez, compiladores de este libro), ya resultaba fructífero desde su actividad investigadora, conocida a través de publicaciones académicas y, especialmente, desde la divulgación que hacen en prensa y redes. Parte de esa tarea divulgadora es el impulso de un espacio académico que permite el debate entre investigadores de todo el mundo, con diversidad de disciplinas, como demuestra el resultado del congreso de 2015 que compila este libro. Con este contexto se comprende mejor la relevancia que tiene esta primera publicación que es reflejo del espíritu plural y el rigor científico que promueve MESO.

El encuentro inaugural, explican los compiladores en el prólogo, da cuenta de la multidimensionalidad que están tomando los estudios sobre medios
T I T U L A R E S,

H A S H A G S

Y VIDEOJ U E G O S

Mitchelstein, E. \& Boczkowski, P. (comps.). (2016). Titulares, hashtags y videojuegos. Buenos Aires: Manantial.

INMEDIACIONES DE LA COMUNICACION 2018 - VOL. 13/N0 1- 299-302-ISSN 1510-5091 - ISSN 1688-8626 (EN LINEA) 
y comunicación, y que puede apreciarse en cinco aspectos: marcos teóricos, métodos, soportes materiales, objetos de estudio y tipos de preguntas. Esta multiplicidad de enfoques configura un índice de trece capítulos que refleja las ponencias seleccionadas entre las cuarenta presentadas a la convocatoria del congreso. No obstante, la pluralidad no impide que la publicación tenga homogeneidad en cuanto a la solidez conceptual y el rigor investigativo de los dieciocho autores de Argentina, Chile, Colombia, Estados Unidos y Francia.

Medios, mercado, instituciones educativas, gobiernos, partidos políticos, comunidades virtuales, ciudadanía son los actores que aparecen en las tramas de comunicación que abordan las partes del libro. El periodismo, las noticias, las tecnologías, la comunicación electoral son los temas abordados desde una variedad de enfoques y métodos que se materializan en computadoras portátiles, videojuegos, publicidades y sitios de noticias.

El académico Silvio Waisbord, de la George Washington University, abrió el congreso y abre el volumen hilvanando todos esos temas en el periodismo, la profesión del campo de la comunicación que más transformaciones está registrando. Más allá de las consabidas crisis, el desafío que describe Waisbord es pensar el lugar que cabe a un actor que detentó muchos años el monopolio de la información y que hacia adelante debe repensar narrativas, soportes y funciones más allá de las conocidas.

En ese sentido, Silvia Ramírez Gelbes, de la UDESA y la Universidad de Buenos Aires (UBA), propone un análisis del discurso noticioso que permite entender la enunciación de los medios digitales, aspecto más relevante en el periodismo contemporáneo que los contenidos de los enunciados. Desde otro acercamiento, Lila Luchessi, dela Universidad Nacional de Río Negro(UNRN), analiza las formas narrativas que adoptan los medios digitales en una localidad argentina. Ambas autoras dan pistas acerca de las formas contemporáneas de producir información. De las transformaciones en la distribución de las noticias se ocupa Víctor García-Perdomo, de la University of Texas. Diseminación, diálogo, promoción son variables que no solían describir el discurso periodístico pero que se vuelven fundamentales para noticias que encuentran en la red social Twitter una continuidad, como demuestran las conclusiones del análisis de las señales CNN en Español, Univisión Noticias, Caracol Noticias y CityTV.

Una de las transformaciones de las organizaciones periodísticas es la especialización en el chequeo de datos que analizan Martín Becerra y Santiago Marino, de la Universidad Nacional de Quilmes (UNQ), a partir de una experiencia argentina. El caso les permite explorar a esos actores emergentes en los intersticios que quedan entre los medios periodísticos ylas organizaciones dela sociedad civil para aportar un eslabón dela cadena informativa. También desde la economía política, una tradición muy afianzada en el continente, Iván Schuliaquer, de la Universidad Nacional de General Sarmiento (UNGS), estudia la relación de las empresas mediáticas de Uruguay con el gobierno uruguayo en 
la década 2005-2015. El mismo diagnóstico del panorama mediático regional, que planteaba como problema central la alta concentración de medios, hizo coincidir a varios países en la solución de encarar reformas legislativas, aunque no necesariamente todos registraron iguales resultados. A los testimonios que describen el derrotero del caso uruguayo, Philip Kitzberger, de la Universidad Di Tella, agrega una sistematización de las reformas en las políticas de medios que tuvieron lugar en toda América Latina. La clasificación muestra diferencias sustanciales en los procesos reformistas al incorporar la variable políticoinstitucional para explicar procesos a la vez similares y disímiles.

Las campañas políticas son abordadas en dos capítulos. Los contenidos de avisos de las campañas publicitarias son descriptos por Natalia Aruguete, de la UNQ, para analizar de quéhablaron los candidatos presidenciales de Argentina en 2011, de México en 2012 y de Venezuela en 2012. Por su parte, los compiladores del libro, Eugenia Mitchelstein y Pablo J. Boczkowski, le dan a la campaña presidencial de 2011 en Argentina un análisis complementario al cruzar la agenda de los medios con la atención del público. Al combinar herramientas metodológicas pueden abordar la complejidad de las instancias electorales y mostrarlas como objetos poliédricos que combinan enunciadores, procesos de circulación y destinatarios que no siempre se convierten en receptores al final del proceso de comunicación.

Los objetos de estudios más recientes están incluidos a través de las tecnologías educativas en el capítulo de Anita Say Chan, de la Universidad de Illinois, que analiza desde la etnografía los foros de Tecnologías de la Información y la Comunicación (TICs) para el desarrollo y la educación en América Latina, con foco en el Perú. Los videojuegos son estudiados desde las teorías sobre la apropiación sociotécnica por los colombianos Enrique Uribe-Jongbloed, de la Universidad del Norte, Hernán D. Espinosa-Medina y Sergio Roncallo-Dow, de la Universidad de La Sabana, Colombia. Fernando Valenzuela, de la Universidad Andrés Bello (UNAB) y Claudio Ramos Zincke, de la Universidad Alberto Hurtado (UAH), Chile, ubican su trabajo entre los estudios de tecnología y las ciencias de la comunicación para dar cuenta del tratamiento informativo del tema de la violencia escolar. Arturo Arriagada, de la Universidad Adolfo Ibáñez, aporta el concepto "capital digital" que analiza a partir del comportamiento de grupos de fans de música independiente en Chile y la utilización que les dan a las tecnologías para desarrollar sus intereses.

La variedad de los contenidos es un indicador positivo de la interdisciplinaridad pero, a la vez, plantea la dificultad que tiene delimitar el campo de la comunicación desde un enfoque dominante. De ahí que los compiladores destaquen en el prólogo que son muy distintas las líneas de investigación que atraviesan los capítulos: la sociología de la educación (Say-Chan; Valenzuela y Ramos-Zincke); los estudios del periodismo (Waisbord); los estudios de las noticias y su consumo (Mitchelstein y Boczkowski); la sociología de la 
profesión (Luchessi); los estudios de las narrativas (García Perdomo; Ramírez Gelbes); la ciencia política (Kitzberguer); la economía política de los medios (Becerra y Marino; Schuliaquer); la comunicación política (Aruguete); y estudios delos nuevos soportes (Arriagada; Uribe-Jongbloed, Espinosa-Medina y Roncallo-Dow).

Muchos capítulos aprovechan las posibilidades de la digitalización de los medios para expandir las muestras y aportar comparaciones amplias que abren el alcance de los análisis y las explicaciones. Al recurso clásico de las entrevistas personales se agregan los foros y las publicaciones en las redes, o bien, se triangulan los métodos de análisis de contenido, encuestas y entrevistas en profundidad. Las técnicas clásicas del siglo pasado se aplican a los objetos de este siglo, o se reinventan en nuevos métodos. En la mayoría de los casos los autores aprovechan el valor didáctico y argumentativo que aporta la visualización de datos en gráficos. Es en este punto que se aprecia la renovación que significa para la tradición ensayística latinoamericana el intercambio con investigadores que vienen con experiencias y prácticas de otros ámbitos académicos. Ese intercambio va dando pruebas de la eficacia de abordar los objetos de estudios latinoamericanos con parámetros científicos globales.

Más allá del valor que tienen individualmente las investigaciones para los especialistas interesados en los distintos temas, el aporte de la publicación en su conjunto es expandir los límites del campo de la comunicación que en el Cono Sur se había vuelto, hacia el fin de siglo, un tanto endogámico y monolítico en cuanto a temas, métodos y perspectivas. El congreso y libro derivado demuestra que hay comunicación más allá de los mensajes: que está en los soportes, en los procesos, en los contextos, en los efectos. La apertura a otras preguntas y a otras disciplinas no siempre consideradas en los congresos locales que representa este primer hito de MESO es en sí misma una instancia renovadora para un campo que se ha llenado de objetos de estudio renovados, que ya no admiten abordajes anquilosados.

\section{IDENTIFICACIÓN DE LAAUTORA}

Adriana Amado es Doctora en Ciencias Sociales porla Facultad Latinoamericana de Ciencias Sociales (Flacso), especializada en temas de comunicación pública y periodismo. Es investigadora y docente en Iberoamérica. Es autora de quince libros de su especialidad, entre los que los últimos títulos son Política pop: de líderes populistas a telepresidentes (2016) y La prensa de la prensa: periodismo y relaciones públicas en la información (2016). Es activista cívica en varias oenegés argentinas dedicadas a la comunicación y la democracia. 